\title{
Comparison of the performance of different radar pulse compression techniques in an incoherent scatter radar measurement
}

\author{
B. Damtie ${ }^{1}$ and M. S. Lehtinen ${ }^{2}$ \\ ${ }^{1}$ Washera Geospace and Radar Science Laboratory, P.O. Box, 79, Bahir Dar University, Bahir Dar, Gojjam, Ethiopia \\ ${ }^{2}$ Sodankylä Geophysical Observatory, Tähteläntie 62, 99600 Sodankylä, University of Oulu, Finland
}

Received: 28 August 2008 - Revised: 10 January 2009 - Accepted: 27 January 2009 - Published: 17 February 2009

\begin{abstract}
Improving an estimate of an incoherent scatter radar signal is vital to provide reliable and unbiased information about the Earth's ionosphere. Thus optimizing the measurement spatial and temporal resolutions has attracted considerable attention. The optimization usually relies on employing different kinds of pulse compression filters in the analysis and a matched filter is perhaps the most widely used one. A mismatched filter has also been used in order to suppress the undesirable sidelobes that appear in the case of matched filtering. Moreover, recently an adaptive pulse compression method, which can be derived based on the minimum mean-square error estimate, has been proposed. In this paper we have investigated the performance of matched, mismatched and adaptive pulse compression methods in terms of the output signal-to-noise ratio (SNR) and the variance and bias of the estimator. This is done by using different types of optimal radar waveforms. It is shown that for the case of low SNR the signal degradation associated to an adaptive filtering is less than that of the mismatched filtering. The SNR loss of both matched and adaptive pulse compression techniques was found to be nearly the same for most of the investigated codes for the case of high SNR. We have shown that the adaptive filtering technique is a compromise between matched and mismatched filtering method when one evaluates its performance in terms of the variance and the bias of the estimator. All the three analysis methods were found to have the same performance when a sidelobe-free matched filter code is employed.
\end{abstract}

Keywords. Radio science (Ionospheric physics; Signal processing; Instruments and techniques)

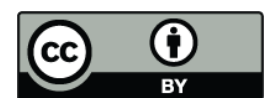

Correspondence to: B. Damtie (bayliedamtie@yahoo.com)

\section{Introduction}

An incoherent scatter radar measures ionospheric parameters like electron density, electron temperature, ion temperature and plasma velocity by transmitting a modulated radio wave into the ionosphere and receiving the scattered signals. The receive signals provide valuable information on the ionosphere. The characteristics of the ionosphere depend on location and important information on the physics of the ionosphere can be obtained from its spatial behavior. Thus an incoherent scatter radar should be capable of measuring the ionosphere over a small range extent depending on the scale heights of the ionosphere. In addition it is important to know the temporal characteristics of the ionosphere, which can provide crucial information on the dynamics of the ionosphere. In order to capture these and other useful information from the ionosphere we need to use an appropriate data acquisition and signal processing method. Especially, one needs to exploit the flexibility of a modern incoherent scatter radar in terms of modulating the transmitter waveform and filtering the received echoes. Moreover, the advent of modern computer technology has made it possible in practice to easily employ different signal processing methods.

Many authors have presented various types of incoherent scatter radar experiments which employ different modulation schemes, data acquisition and analysis methods. Typical modulations cited in various publications include single pulses and multipulses (Farley, 1969, 1972), random codes (Sulzer, 1986) and alternating codes (Lehtinen and Häggström, 1987). Usually a very high spatial resolution incoherent scatter radar measurement is obtained by employing a Barker coded (Barker, 1953) transmission and then filtering the received signals by a matched filter. Several researchers, including Ioannidis and Farley (1972), Turunen et al. (1985), Wannberg (1993), and Lehtinen et al. (2002) have presented different types of incoherent scatter radar experiments by arranging Barked-coded transmissions in various manners.

Published by Copernicus Publications on behalf of the European Geosciences Union. 


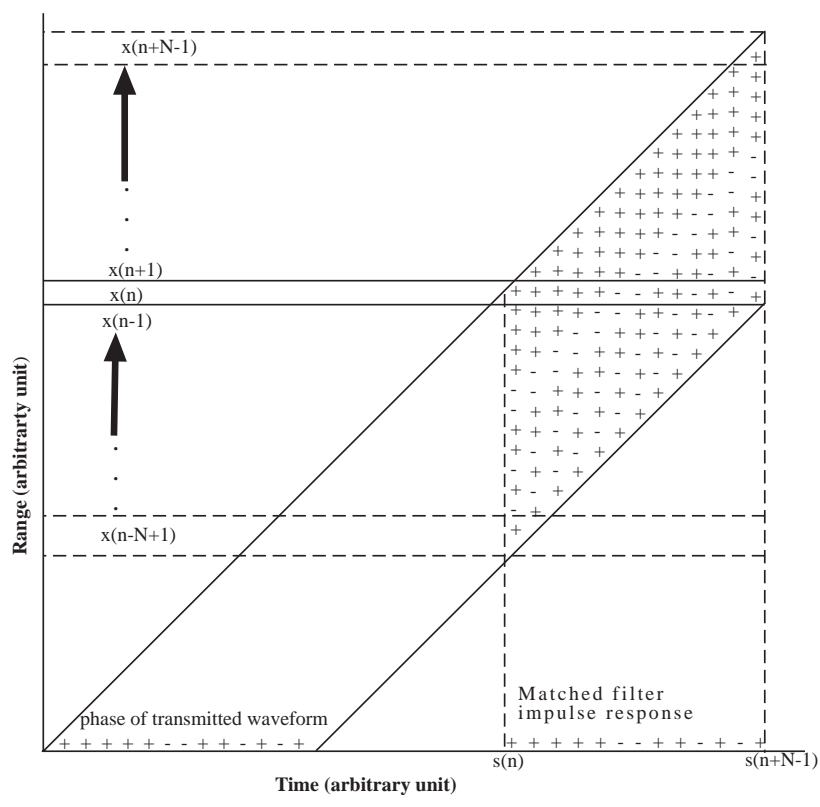

Fig. 1. Schematic diagram that shows the spatial and temporal projections of a sample at the output of the matched filter.

Decoding of Barker-coded incoherent scatter radar measurements is usually carried out in amplitude domain by employing a filter, which has an impulse response of the inverse replica of the corresponding transmitted waveform. In this arrangement we can see that each individual sample at the output of the matched filter equals the weighted sum of the impulse response of the ionosphere illuminated by the transmission in a time duration limited by the bandwidth of the filter. One usually considers the impulse response of the ionosphere at a specific gate to be stationary during the passage of the transmitted pulse through it. Based on this assumption it is easy to see that each individual sample at the output of the matched filter is mainly the weighted sum of the impulse response of the ionosphere from a specific nominal range gate. However, we also get unwanted contributions from neighbouring non-nominal range gates, depending on the nature of the ionosphere.

Different methods of eliminating these unwanted contributions in Barker-coded transmission and other binary phase radar waveforms have been presented. Key et al. (1959) have used weighting networks that are placed after the standard matched filter, which reduces the sidelobes to an arbitrary low level. A method of eliminating the sidelobes in periodic binary phase codes by means of mismatched filtering has been presented by Rohling and Plagge (1989). However, this mismatched filtering and the references within has an associated SNR losses. Binary phase codes that give minimum possible SNR losses when filtered with their aperiodic inverse filters are available in Rupretcht and Rupf (1996).

The present authors have carried out an exhaustive computer search of optimal binary phase code-mismatched filter pairs up to a code length of 25 and the advantages of eliminating the sidelobes in the analysis of an incoherent scatter measurement were demonstrated in practice by Lehtinen et al. (2004) and the reference within. The present authors have also extended these kinds of investigations to aperiodic quadriphase codes Damtie et al. (2008). In all these works it is shown that a mismatched filter can have a substantial SNR losses in the case of some binary radar waveforms. Recently, have proposed that adaptive filtering technique can also be used to carry out pulse compression without creating unwanted sidelobes.

The purpose of this paper is to compare the performance of the matched, mismatched and adaptive pulse compression methods by considering different types of optimal codes and also for different SNR values. This is carried out by using a metric that includes the output SNR and the bias and variance of the estimator.

\section{Review of different radar pulse compression tech- niques}

We shall base our discussion on a pulsed incoherent scatter radar measurement and also consider a discrete real signal. Each individual signal $s(n)$ of the radar echo that reaches the receiver at a particular discrete time index $n$ may be described by

$s(n)=\sum_{k=0}^{N-1} x(n-k) p(k)+\xi(n)$,

where $x(n-k)$ is a random variable that represents scatter amplitude from a single pulse, $p(k)$ is the transmitted radar waveform (the high frequency carrier signal is excluded in this formulation without any loss of information for our purpose) and $\xi(n)$ is the additive noise. In this work the index $n$ is equivalent to $n$ times the sampling time interval and it can be translated into range. It is easy to see that each individual receive signal $s(n)$ contains contributions from different range gates (i.e. $x(n), x(n-1), \ldots, x(n-N+1)$ ), where $N$ denotes the length of the transmitted code.

It is common to use a suitable filter to enhance the output SNR. In other words the signal described by Eq. (1) should be decoded by using an appropriate decoding filter. If we denote the impulse response of this filter by $h(n)$ the filtered signal, denoted here by $y(n)$, at the output is commonly described by (see for example a textbook by Hayes (1996))

$y(n)=\sum_{k^{\prime}=0}^{N-1} h\left(k^{\prime}\right) s\left(n-k^{\prime}\right)$.

Here we have considered that the length of the decoding filter is equal to the transmission waveform and this means that the filter coefficients are $h(0), h(1), \ldots, h(N-1)$. Also, the index is chosen to make $y(n)$ to correspond to the nominal range gate, which gets maximum illumination time during the passage of the transmitted pulse. 

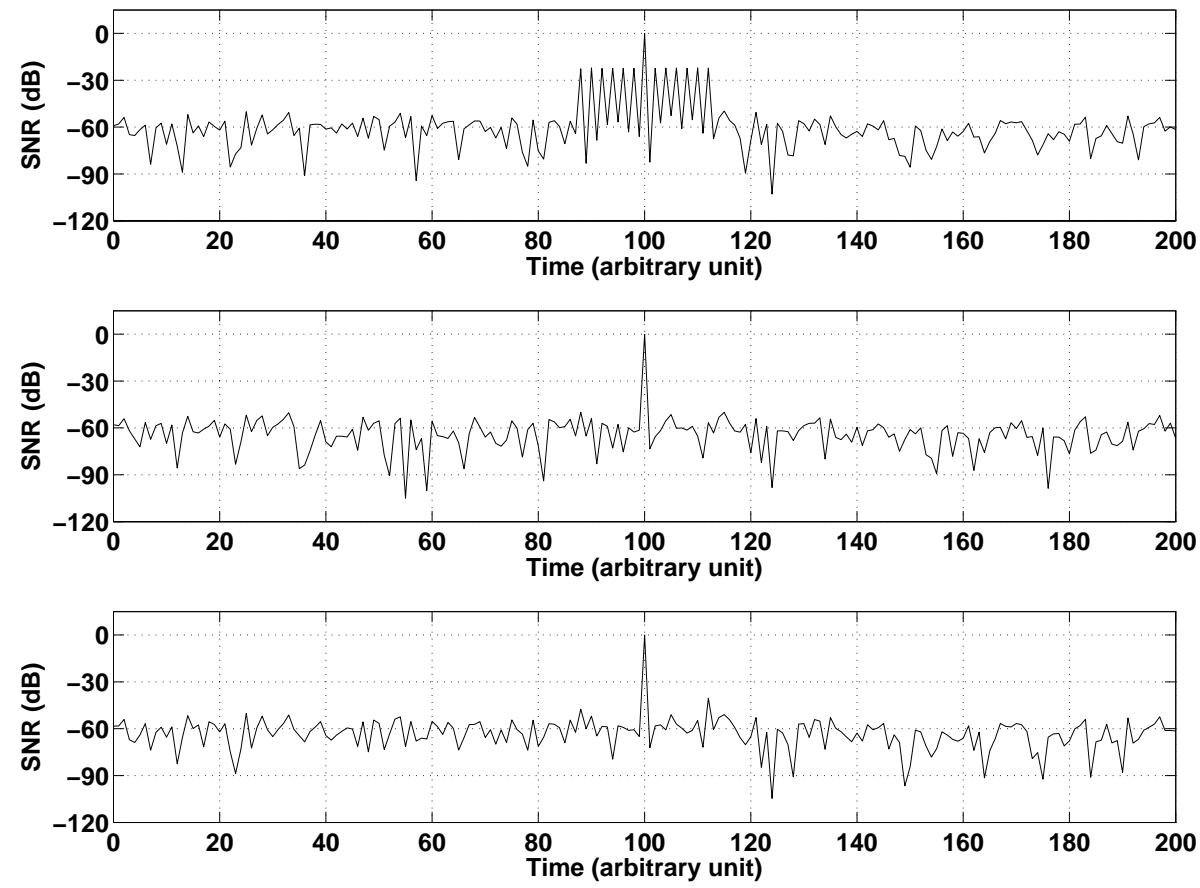

Fig. 2. Output signal waveforms from different radar pulse compression techniques; top panel: matched filter output, middle panel: mismatched filter output, and bottom panel: adaptive filter output.

\subsection{Matched and mismatched pulse compression tech- niques}

Matched filtering is perhaps the most popular and widely used filtering method in a radar system. The principle is describe in many publications including a textbook by Clarkson (1993) and the reference therein. The impulse response of a matched filter, denoted here by $h_{m}(n)$, is simply the inverse replica of the transmitted waveform and that is

$h_{m}(n)=p(-n)$.

For comparison purpose, we illustrate how a matched filter works by considering a simplified incoherent scatter measurement scenario. The schematic in Fig. 1 shows how a particular sample at the output of a filter that matches the transmission can be projected into the spatial and temporal extension of the ionosphere. In other words it depicts how the signals $s(n), s(n+1), \ldots, s(n+N-1)$ in the right side of Eq. (2) are related to the true impulse response of the target at different range gates (i.e. $x(n), x(n-1), \ldots, x(n-N+1)$ ) contained in Eq. (1). In this kind of arrangement we can see that the output signal mainly comes from the nominal range gate $x(n)$ when we assume that the signal does not change significantly during the passage of the transmitted pulse through it. This can be clearly seen in Fig. 1 from the phase patterns of the transmission at different range gates in conjunction with the impulse response of the receiver. However, there is also a small contribution from the non-nominal range gates depending on the nature of the transmission waveform. For example, for the 13-bit Barker code transmission in Fig. 1 one can readily identify that there will be a small contribution from $x(n-N+1), x(n-N+3), \ldots, x(n+N-1)$.

Mismatched filtering technique is a method of pulse compression that does not produce unwanted sidelobes. An appropriate mismatched filter can be constructed by exploiting the characteristics of the transmission waveform as presented in Damtie et al. (2008). Its impulse response, denoted here by $\lambda_{\text {mis }}(n)$ is given by

$\lambda_{\text {mis }}(n)=\lim _{M \rightarrow \infty} \frac{1}{M} \sum_{k=0}^{M-1} \frac{N}{\hat{\mathbf{P}}(k)} e^{-i 2 \pi n k}$,

where $\hat{\mathbf{P}}(k)$ and $N / \hat{\mathbf{P}}(k)$ are the discrete Fourier transforms of $p(n)$ and $\lambda_{\text {mis }}(n)$, respectively, and $M$ is the length of the discrete Fourier transform.

\subsection{Adaptive pulse compression technique}

An adaptive signal processing is a widely known signal processing technique and it has been used in various applications. The general theory of adaptive signal processing is old and it can be obtained from various publications including the widely cited textbook by Widrow and Stearns (1985). Many researchers have showed the applications of adaptive signal processing in a radar system. In the case of incoherent scatter radar measurement Wen et al. (2005) have used adaptive filtering to separate an incoherent scatter signal from a meteor echo. This has been done by determining the impulse 

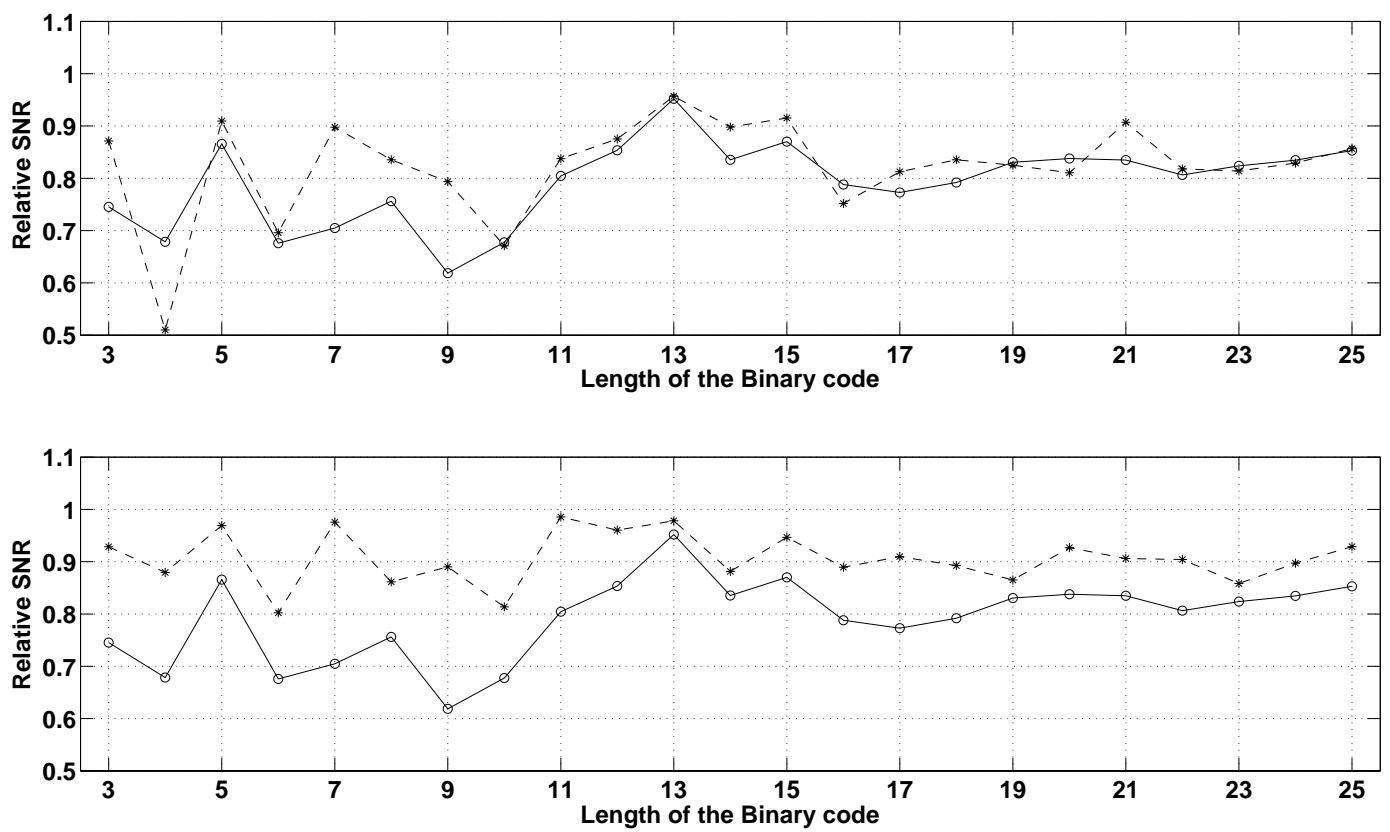

Fig. 3. SNR losses associated to the mismatched $\left(R_{\text {mis }}\right)$ and adaptive $\left(R_{a}\right)$ pulse compression methods; top panel (for the case of high SNR): mismatched filtering (solid line with o), adaptive filtering (broken line with *), bottom panel (for the case of low SNR): mismatched filtering (solid line with o), adaptive filtering (broken line with *).

response of an appropriate filter in accordance with the nature of the scattered signal. These authors have used a filterbank to facilitate the analysis in practice. Recently a comprehensive presentation of an adaptive radar signal processing has been published in a textbook by Haykin (2007).

Radar pulse compression by means of adaptive signal processing has been proposed recently as another alternative to suppress the unwanted sidelobes in the analysis of a radar measurement. and the reference therein have showed how to estimate the impulse response of a radar target using an adaptive pulse compression technique. Their predictive deconvolution approach is based on the minimum mean square error (MMSE) estimation theory as presented in a textbook by Kay (1993).

The basic idea of the minimum mean square error (MMSE) adaptive pulse compression method is to replace $h\left(n-k^{\prime}\right)$ in Eq. (2) by $w_{a}\left(n-k^{\prime}\right)$ whose filter coefficients varies in accordance with the characteristics of the input signals $s(n), s(n+1), \ldots, s(n+N-1)$. This means that a filter with different impulse response is used to obtain the estimate of each range gate. The mathematical formulation of this kind of filter can be simplified using matrix notation. By considering the impulse response of the target at different range gates to be uncorrelated to each other (i.e. $E\left(x(n) x\left(n^{\prime}\right)\right)=0$, for $\left.n \neq n^{\prime}\right)$ and also the background noise to be white, the impulse response of the adaptive filter, which gives the estimate of $x(n)$, can be expressed by (see for detailed mathematical derivation)
$\mathbf{w}_{a}^{n}=\rho(n)\left(\mathbf{C}_{n}+\sigma^{2} \mathbf{I}\right)^{-1} \mathbf{P}$,

where $\sigma^{2}$ denotes the noise power, $\rho(n)=E\left(x^{2}(n)\right)$ is the average power of $x(n), \mathbf{P}=\{p(0), p(1), \ldots, p(N-1)\}$ represents the transmission waveform, $\mathbf{I}$ is an identity matrix, $\quad \mathbf{C}_{n}=\sum_{n^{\prime}=-N+1}^{N-1} \rho\left(n+n^{\prime}\right) \mathbf{P}_{n^{\prime}} \mathbf{P}_{n^{\prime}}^{T} \quad$ and $\mathbf{w}_{a}^{n}=\left\{w_{a}^{n}(0), w_{a}^{n}(1), \ldots, w_{a}^{n}(N-1)\right\} . \quad$ Here $\mathbf{P}_{n^{\prime}}$ contains the elements of the transmitted waveform shifted by $n^{\prime}$ samples and the remainder zero-filled and $T$ is the transpose. One can see from Eq. (5) that the values of the elements of $\mathbf{w}_{a}^{n}$ are calculated from the noise power and using an initial estimate of the scattered signal power. The adaptive filtering is carried out in an iterative manner and its convergence can be faster by making use of an optimized initial signal power estimator. The algorithm for an adaptive filtering pulse compression method is nicely illustrated by and for our purpose we can summarize the technique in the following manner:

1. Obtain an initial estimate of the signal power $\rho(n)$ for all range gates from the sample sequence $s(1), s(2), \ldots, s(m)$ using an optimal power estimator.

2. Estimate the associated noise power $\sigma^{2}$.

3. Calculate the corresponding $\mathbf{w}_{a}^{n}$ using Eq. (5) for each range gate.

4. Carry out the adaptive filtering operation to obtain the estimate of the signal at different range gates. 

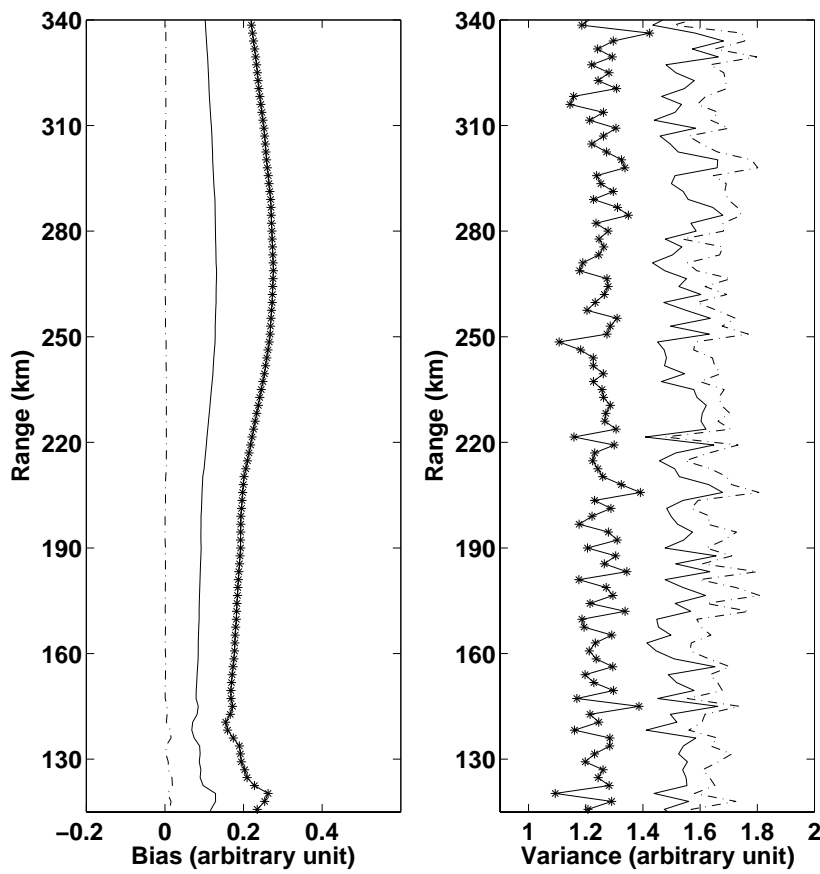

Fig. 4. Bias and variance of different estimators for the case of high SNR; left panel: the bias of the matched filter estimator (solid line with *), the bias of the mismatched filter estimator (broken line), the bias of the adaptive filter estimator (solid line), right panel: the variance of the matched filter estimator (line with *), the variance of the mismatched filter estimator (broken line), the variance of the adaptive filter estimator (solid line).

5. Using the output obtained in stage 4, repeat the calculation starting from stage 3 and this process may continue until suitable convergence is obtained.

It is useful for this review to demonstrate the three types of pulse compression methods by simulating a scattering signal from a stationary point like radar target in the presence of white noise. We have simulated the receive signal profile at the output of each pulse compression filter by using a measurement scenario as shown in Fig. 1. It is well known that the scattered signal waveform from such a target takes the shape of the transmission pulse with some disturbances by noise. We considered a 13-bit Barker coded transmission in the simulation and the noise power is considered to be $60 \mathrm{~dB}$ lower than the signal power (high SNR case).

Decoding the scattered signal using the matched filter yields the result shown in the top panel of Fig. 2, which has unwanted sidelobes with magnitude of about $30 \mathrm{~dB}$ higher than the background noise power. The magnitude of these sidelobes can even be stronger for some other kinds of less optimal codes including the widely used 5-bit Barker code. The middle and lower panels of Fig. 2 depict the output waveforms obtained by means of mismatched and adaptive filtering techniques, respectively. One can see that the unwanted sidelobes created in the case of matched filtering have
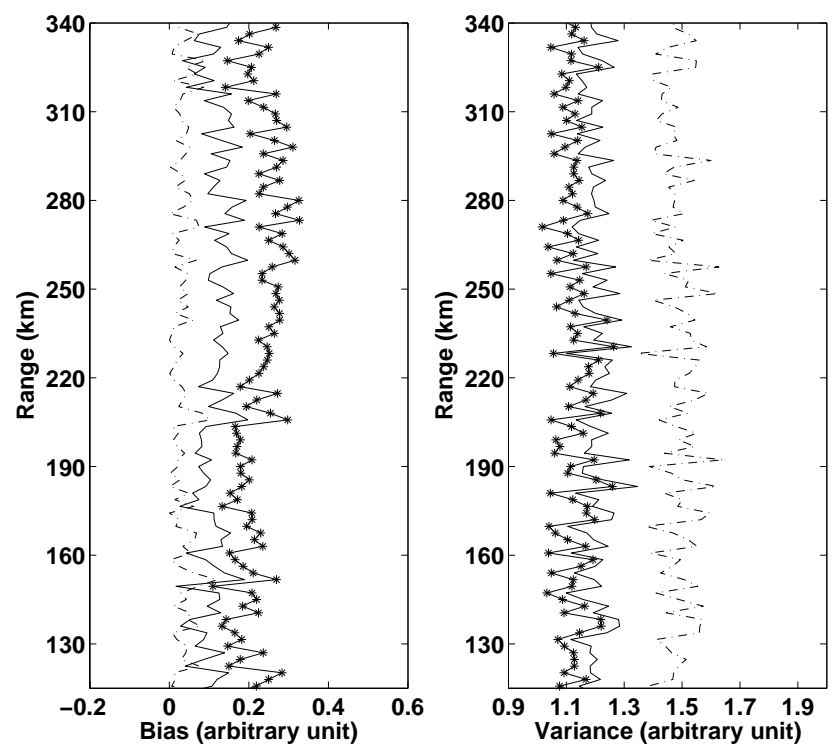

Fig. 5. Bias and variance of different estimators for the case of low SNR; left panel: the bias of the matched filter estimator (solid line with $*)$, the bias of the mismatched filter estimator (broken line), the bias of the adaptive filter estimator (solid line), right panel: the variance of the matched filter estimator (line with *), the variance of the mismatched filter estimator (broken line), the variance of the adaptive filter estimator (solid line).

been suppressed to the noise level in both cases. The adaptive filtering result was obtained by using two stage iterations and it was found to be enough to get the required convergence. In this investigation we shall implement an adaptive filtering using two stage iterations and found that in most cases it is enough.

\section{SNR performance comparison of adaptive and mis- matched pulse compression techniques}

We have seen that one may use use an adaptive or a mismatched filter when the effects of the sidelobes in the case of a matched filter is a concern. In this section we investigate which one of these two techniques gives less degradation in the output SNR. It is well know that a matched filter gives the maximum possible output SNR for a stationary point target in the presence of white noise. Thus it is often used as a reference to measure the performance of other types of filters. For example, Rupretcht and Rupf (1996) and Damtie et al. (2008) have used it as a reference to quantify the performance of different types of radar waveform and filter pairs. A similar approach is used in this work. This means that we carry out the SNR performance comparison of the adaptive and the mismatched pulse compression techniques by calculating the relative output SNR (relative to the output SNR from the corresponding matched filter). This is done by considering a scattered signal from a stationary point target in 

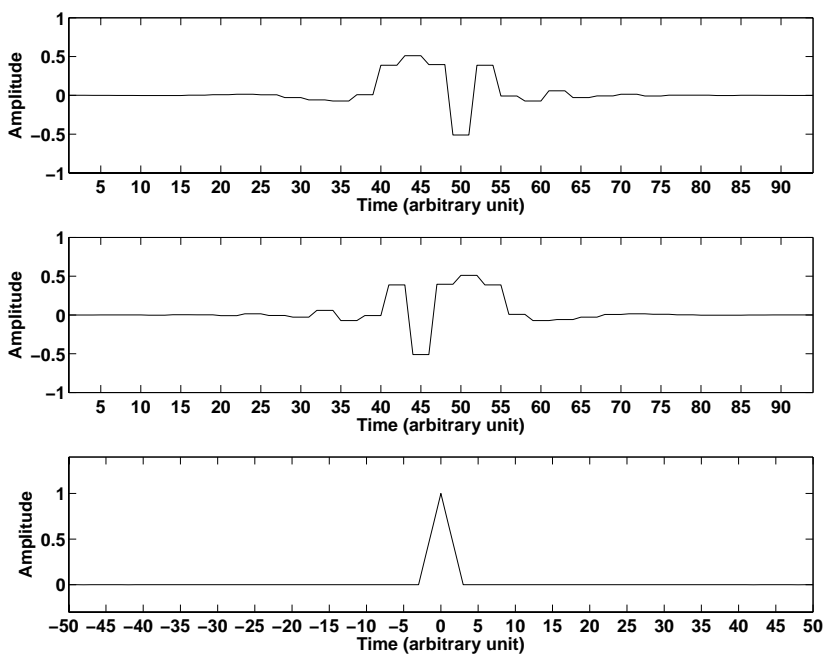

Fig. 6. Illustrations of the sidelobe-free matched filter code; top panel: the sidelobe-free matched filter code, middle panel: the impulse response of the associated matched filter, and bottom panel: the convolution result.

the presence of white noise. Then the ratio of the output SNR from the mismatched or adaptive filter to the corresponding output from the matched filter is calculated at the instant of the point target detection.

This ratio for the case of a mismatched filter with an impulse response of $\lambda_{\text {mis }}(n)$ is given by (see Lehtinen et al. (2004) for details)

$R_{\mathrm{mis}}=\sum_{n=0}^{N-1}\left|h_{m}(n)\right|^{2}\left(\sum_{n=-\infty}^{\infty}\left|\lambda_{m i s}(n)\right|^{2}\right)^{-1}$,

where $R_{\text {mis }}$ denotes the ratio of the output SNR of a mismatched filter to the corresponding output from a matched filter. Similarly, we can have

$R_{a}=\sum_{n=0}^{N-1}\left|h_{m}(n)\right|^{2}\left(\sum_{n=0}^{N-1}\left|w_{a}^{n}(n)\right|^{2}\right)^{-1}$,

where $R_{a}$ is the ratio of the output SNR of an adaptive filter to that of the corresponding matched filter. We can now investigate the values of $R_{\mathrm{mis}}$ and $R_{a}$ by taking different types of radar waveforms and in the present paper we use the optimal binary phase codes presented in Damtie et al. (2008).

The top panel of Fig. 3 shows the values of $R_{\mathrm{mis}}$ and $R_{a}$ (the SNR losses associated to the adaptive and mismatched filtering methods, respectively) for the case of high SNR and one can see that they in general have similar losses for most of the binary codes. The 4-bit binary code with phase sequence of $[1,-1,-1,-1]$ has produced the largest degradation in case of adaptive filtering method. Similarly, the performance of these two pulse compression methods for the case of low SNR is portrayed in the bottom panel of Fig. 3 and in this case the adaptive filtering technique has less SNR losses than that of the mismatched filtering for all the binary sequences investigated in here. For most of the codes adaptive filtering has about 10 percent SNR losses compared to the more than 10 percent SNR losses for the case of mismatched filtering. This means that adaptive filtering technique outperforms mismatched filtering for the case of low SNR. This result is in agreement with the basic principle of an adaptive filter design and that is minimizing both the variance and the bias of the corresponding estimator. It is interesting to see that the 13-bit Barker code is the optimal code in both cases having about 5 percent SNR losses. Also, its performance is not affected appreciably by the level of the input SNR.

\section{Investigations of bias and variance in pulse compres- sion: for the case of ordinary radar waveforms}

We know that there is a bias associated with the matched filter estimate due to the sidelobes. The adaptive filter estimator may be understood as an attempt to minimize the bias of the matched filter with a small degradation in the output SNR. There is no bias in the case of the mismatched filter estimator except the degradation of the output SNR. In this section we carry out numerical investigations to compare the performance the three kinds of pulse compression methods in terms of the bias and variance of the estimator by taking different levels of SNR.

The analysis is carried out by considering an incoherent scatter radar signal from a model ionosphere described by Alcaydé et al. (1994) and an arbitrarily chosen 8-bit optimal binary phase transmission waveform with phase sequence $[1,-1,1,-1,-1,1,1,1]$. The receive signal profile at the output of the receiver has been simulated by employing the incoherent scatter radar measurement theory presented in Lehtinen et al. (1996). And then all the three pulse compression methods were utilized to estimate the scattered signal imbedded in the white noise.

Figure 4 shows the bias (left panel) and the variance (right panel) associated to the matched, mismatched and adaptive pulse compression techniques for the case of high SNR. The results clearly portray that the bias of the adaptive filter estimator is less than the corresponding bias of the matched filter estimator. As expected there is no bias associated to the mismatched filter estimator. However, the mismatched filtering technique has produced the largest variance as shown in the left panel. The minimum variance is obtained by means of matched filtering. It is clear to see that the adaptive filtering technique gives some sort of compromised performance between the matched and mismatched filtering methods in terms of bias and variance.

In a similar manner Fig. 5 portrays the bias (left panel) and the variance (right panel) associated to the matched, mismatched and adaptive pulse compression techniques for the case of low SNR. In this case the adaptive filtering technique 

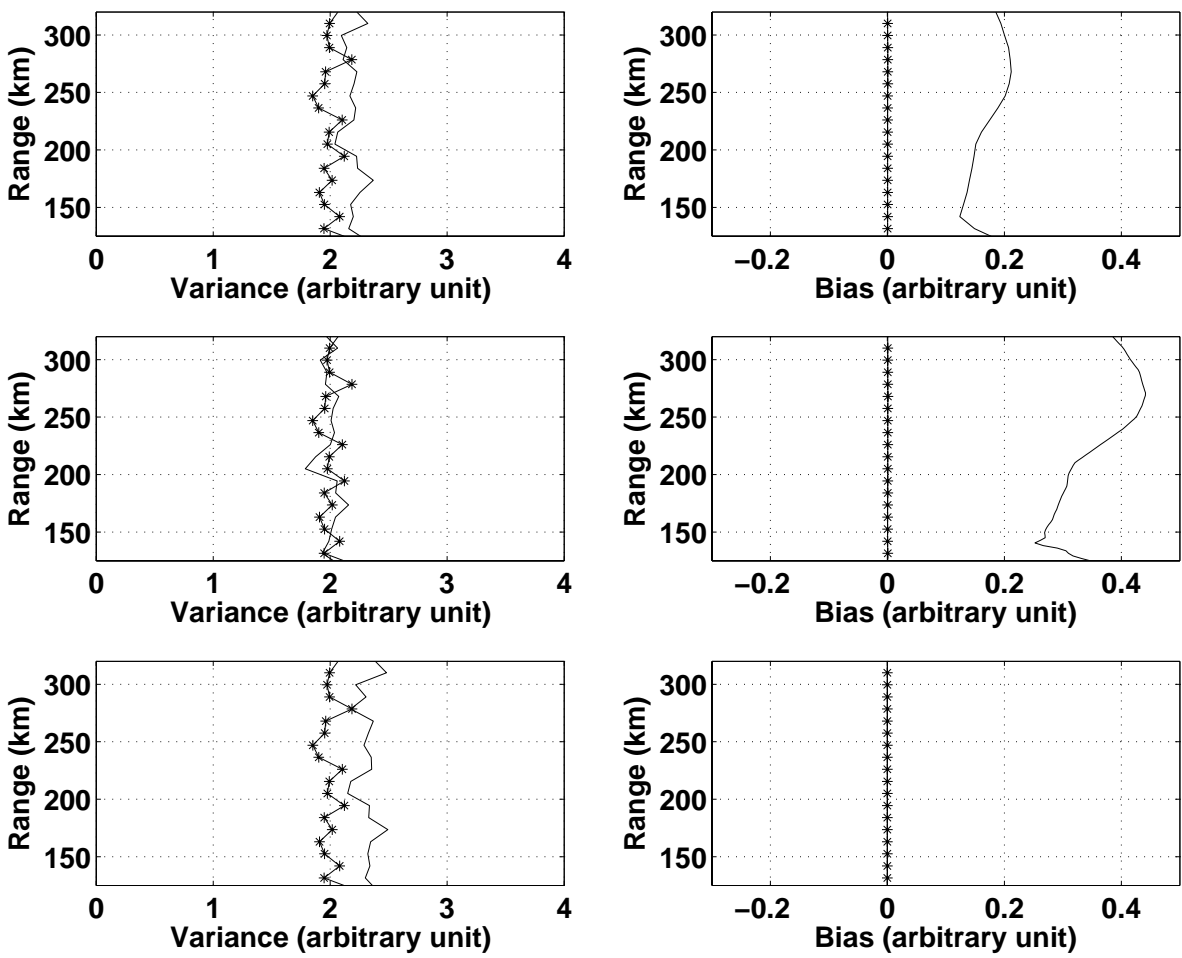

Fig. 7. Bias and variance of different estimators for the case of the sidelobe-free matched filter code (solid line with *) and the 5-bit Barker code (solid line) for high SNR; left panel: variance of the adaptive filter estimator (top panel), variance of the matched filter estimator (middle panel), and variance of the matched filter estimator (bottom panel); right panel: bias of the adaptive filter estimator (top panel), bias of the matched filter estimator (middle panel), and bias of the mismatched filter estimator (bottom panel).

has significantly reduced the variance of the estimator when compared to that of the mismatched filter estimator. Also, one can see that the associated bias is not very large. Hence, an adaptive filter becomes a very good compromise in this case when one wants to suppress the sidelobes without having large variance.

\section{Investigations of bias and variance in pulse compres- sion: for the case of sidelobe-free matched filtering codes}

It is shown that the matched pulse compression technique creates the largest bias and the smallest variance when compared with the other two pulse compression methods in both high and low SNR cases. This means that a matched filter that does not create sidelobes is needed to obtain optimal results in terms of both variance and bias. Then one may wonder if it is possible to find codes that do not create sidelobes when they are convolved with the associated matched filter. Such codes will not only eliminate the bias but also give the minimum variance just by using the matched filter. Codes with these properties, which we shall call them here sidelobe-free matched filter codes, have been found recently and the method of designing these types of codes shall be presented in a separate paper.

Figure 6 demonstrates the waveform of the sidelobe-free matched filter code (top panel) derived from a 5-bit Barker code, the associate matched filter (middle panel) and the convolution result (bottom panel). One can see that the amplitude of the code in the top panel goes to zero after a certain duration and the output from the matched filter does not contain any sidelobes. This result is exactly the result one will get by means of mismatched filtering as well. In other words the impulse response of the matched and the mismatched filter becomes exactly the same. But what does happen to the impulse response of the corresponding adaptive filters at different range gates for these types of codes? It turned out that the impulse response of the adaptive filter at different range gates also becomes the same as that of the matched filter. This means that the three pulse compression methods merge together.

Numerical investigation similar to the presentation in section 4 has been carried out using 5-bit Barker code and the sidelobe-free matched filter code derived from it. Figure 7 shows the variance (left panel) and the bias (right panel) associated to the three types of pulse compression techniques using these two codes for the case of high SNR. One can see that all the three types of pulse compression methods 

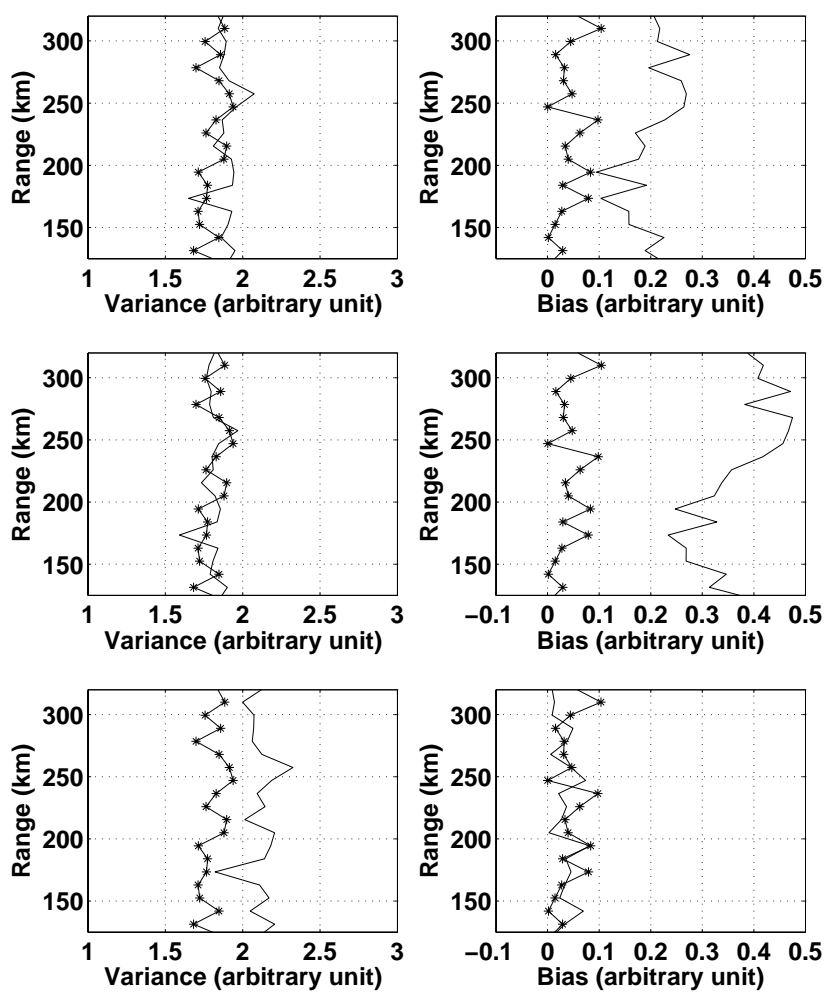

Fig. 8. Bias and variance of different estimators for the case of the sidelobe-free matched filter code (solid line with *) and the 5bit Barker code (solid line) for low SNR; left panel: variance of the adaptive filter estimator (top panel), variance of the matched filter estimator (middle panel), and variance of the matched filter estimator (bottom panel); right panel : bias of the adaptive filter estimator (top panel), bias of the matched filter estimator (middle panel), and bias of the mismatched filter estimator (bottom panel).

have the same performance in terms of both bias and variance in the case of the sidelobe-free matched filtering code. Also, they give better performance by using the sidelobefree matched filter code instead of the corresponding ordinary code. Note that due to randomness of the noise amplitude created at different times, we see small fluctuations in the curves. Also, the variance obtained by matched filtering using ordinary code and sidelobe-free matched filter code is not clearly separated. The bias has been eliminated by using all the three filtering methods when one employes the sidelobe-free matched filter code. Regardless of the type of radar waveform and target, there is no bias in the case of mismatched filtering technique. As result the bias curves obtained from the sidelobe-free matched filter code and ordinary code overlap each other and become hard to differentiate as shown in the bottom left panel of Fig. 7.

Similar bias and variance performance results are obtained for the case of low SNR as shown in Fig. 8. All the three pulse compression methods produce the same variance and there is no bias when the sidelobe-free matched filter code is used even though we see some departure from the exact value due to small integration time. The sidelobe-free matched filter code and the corresponding ordinary 5-bit Barker code yield nearly the same variance in case of matched filtering for both high and low SNR. We can conclude that the sidelobefree matched filter code gives the optimal performance by employing any one of the three pulse compression methods and choice can be made based on computational and implementation simplicity.

\section{Demonstration of the adaptive filtering method in an incoherent scatter radar measurement}

The signal profile portrayed in the left panel of Fig. 9 shows simulated incoherent scatter radar measurement obtained by using 13-bit Barker coded transmission and a model ionosphere described by Alcaydé et al. (1994). The signal was intentionally enhanced around $107 \mathrm{~km}$ to mimic closely located multiple sporadic-E layers and also around $122 \mathrm{~km}$ altitude for stationary point targets. The waveform displayed in the middle panel of Fig. 9 shows the signal profile at the output of the matched filter. We see two main peaks; the first peak, which is relatively wide, denotes the simulated sporadic-E layer and the second peak around $122 \mathrm{~km}$ is from a point target. The sidelobes are also clearly visible in addition to these two main peaks. The main peaks have been cut off to make the horizontal scale suitable for showing the detailed shapes of the sidelobes.

The result from the adaptive filtering method is displayed on the right panel of Fig. 9. One can see that the method is capable of discerning a target, which was masked in the case of matched filtering. We now see two clearly separate peaks; the peaks around $105 \mathrm{~km}$ show multiple sporadic-E layers and the others around $122 \mathrm{~km}$ indicate two point targets. This simple demonstration depicts that the adaptive filtering method can be used when one faces with a problem of separating signals merged together due to the effects of the sidelobes in matched filtering.

\section{Conclusions}

Our knowledge of the Earth's ionosphere has been continuously refined based on in-situ and ground-based measurements. An incoherent scatter radar is one of the most valuable ground-based instruments that has been used extensively for such purpose. It can measure different ionospheric parameters. As result significant efforts have gone into the development of an incoherent scatter radar data acquisition and analysis methods. Initially hardware based data analysis techniques have been replaced by means of software and thereby providing a very flexible environment to implement different modulation schemes and signal processing methods. It is now very common to carry out an incoherent scatter radar signal processing via software instead of using 

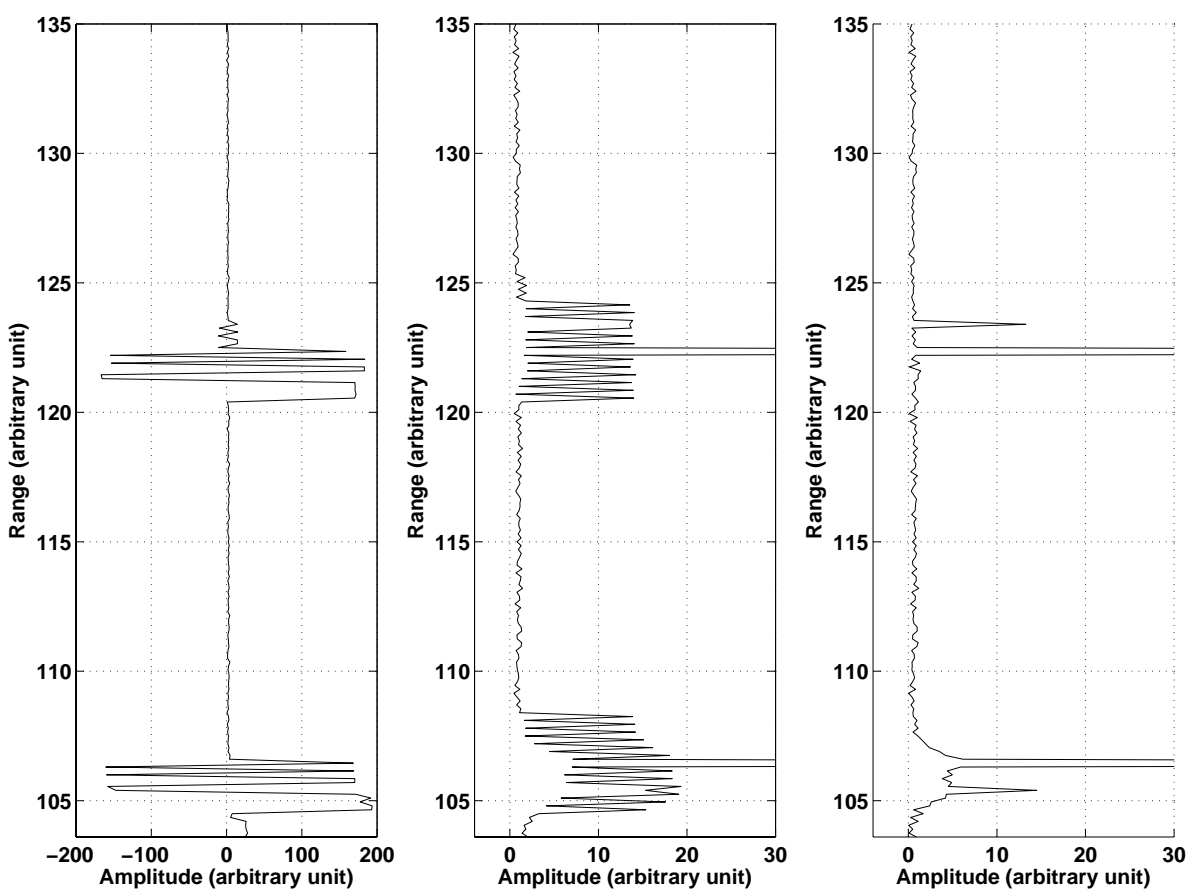

Fig. 9. Demonstration of the adaptive filtering method in the case of an incoherent scatter measurement (left panel: simulated receive signal profile using 13-bit Barker code transmission, middle panel: output signal from the matched filter, right panel: output signal from the adaptive filter).

specialized hardware. This is done by taking the IF stage of the received signal into a system that can be used as a down converter, a low pass filter, an AD converter, a quadrature detector and carry out the sampling and store the resulting samples on hard disk as described in Lehtinen et al. (2002), for example. A similar data acquisition system has been also published by Holt et al. (2000).

The raw data collected in this manner can be analyzed in various manner. For example, a combination of mismatched filtering and lag profile inversion (Damtie et al., 2004) have been used to improve the estimate of an incoherent scatter radar signal. These methods have their pros and cons. The mismatched filtering technique has a small degradation in SNR when compared to the matched filtering method as we have shown. Recently the adaptive filtering method has been proposed as another alternative for radar pulse compression.

In this paper we have compared the performance of the matched, mismatched and adaptive pulse compression methods by using different kinds of optimal codes at various levels of input SNR. We have found that the SNR losses associated to a mismatched filter is greater than an adaptive filter for the case of low SNR input. However, for the case of high SNR these two sidelobe free pulse compression methods cause similar degradation in the output signal. An adaptive filter estimator was found to be a compromise between the matched and mismatched filter estimators when one measures its performance in terms of the associated variance and bias. The impulse response of a matched, mismatched and an adaptive filter turned out to be exactly the same for the case of sidelobe-free matched filter codes for both high and low SNR. As a result it is shown that all the three pulse compression methods have same performance in terms of both variance and bias. In addition, they were found to have better performance when one employs sidelobe-free matched filter code instead of the corresponding ordinary code.

Acknowledgements. This work was supported by the Academy of Finland (application number 213476, Finnish Programme for Centres of Excellence in Research 2006-2011).

Topical Editor M. Pinnock thanks J. Holt and another anonymous referee for their help in evaluating this paper.

\section{References}

Alcaydé, D., Blelly, P.-L., and Lilensten, J.: GIVEME, "General Ionopshere Visualization and Extraction from a Model for the Eiscat Svalbard Radar”, Eiscat Technical Note, 94/52, EISCAT Scientific Association, Kiruna, Sweden, 1994.

Barker, R. H.: Group synchronizing of binary digital systems:, in: Communications Theory, edited by: Jackson, W., Academic, New York, 273-287, 1953.

Blunt, S. D. and Gerlach, K.: Adaptive Pulse Compression via MMSE Estimation, IEEE Trans. Aerospace and Electronic Systems., 42, 572-584, 2006.

Clarkson, P. M.: Optimal and adaptive signal processing, CRC Press, Inc., Florida, 1993. 
Damtie, B., Lehtinen, M. S., and Nygrén, T.: Decoding of Barkercoded incoherent scatter measurements by means ofmathematical inversion, Ann. Geophys., 22, 3-13, 2004, http://www.ann-geophys.net/22/3/2004/.

Damtie, B., Lehtinen, M. S., Orispää, M., and Vierinen, J.: Mismatched Filtering of Aperiodic Quadriphase Codes, IEEE Trans. Inf. Theory, 42, 1742-1749, 2008.

Farley, T. D.: Incoherent scatter correlation measurements, Radio Sci., 4, 935-953, 1969.

Farley, T. D.: Multiple-pulse incoherent-scatter correlation function measurements, Radio Sci., 7, 661-666, 1972.

Hayes, M. H.: Statistical digital signal processing and modeling, John Wiley and Sons, Inc., New York, 1996.

Haykin, S.: Adaptive radar signal processing, John Wiley and Sons, Inc., New Jersey, 2007.

Holt, J. M., Erickson, P. J., Gorczyca, A. M., and Grydeland, T.: MIDAS-W: a workstation-based incoherent scatter radar data acquisition system, Ann. Geophys., 18, 1231-1241, 2000, http://www.ann-geophys.net/18/1231/2000/.

Ioannidis, G. and Farley, D. T.: Incoherent scatter observations at Arecibo using compressed pulses, Radio Sci., 7, 763-766, 1972.

Kay, S. M.: Fundamentals of statistical signal processing: estimation theory, Upper Saddle River, NJ: Prentice-Hall, 1993.

Key, E. L., Fowle, E. N., and Haggarty, R. D.: A method of sidelobe suppression in phase coded pulse compression systems, MIT, Lincoln Laboratory, Report No. Tech Report, 209, November 1959.

Lehtinen, M. S. and Huuskonen, A.: General incoherent scatter analysis and GUISDAP, J. Atmos. Terr. Phys., 58, 435-452, 1996.

Lehtinen, M. S., Markkanen, J., Väänänen, A., Huuskonen, A., Damtie, B., Nygrén, T., and Rakola, J.: A new incoherent scatter technique in the EISCAT Svalbard Radar, Radio. Sci., 37(4), 1050, doi:10.1029/2001RS002518, 2002.
Lehtinen, M. S., Damtie, B., and Nygrén, T.: Optimal binary phase codes and sidelobe-free decoding filters with application to incoherent scatter radar, Ann. Geophys., 22, 1623-1632, 2004, http://www.ann-geophys.net/22/1623/2004/.

Lehtinen, M. S. and Häggström, I.: A new modulation principle for incoherent scatter measurements, Radio Sci., 22, 625-634, 1987.

Sulzer, M. P.: A radar technique for high range resolution incoherent scatter autocorrelation function measurements utilizing the full power of the klystron radars Radio Sci., 21, 1035-1040, 1986.

Rohling, H. and Plagge, W.: Mismatched-filter design for periodical binary phased signals, IEEE Trans. Aerospace and Electron. Syst., 25, 890-896, 1989.

Rupretcht, J. and Rupf, M.: On the search for Good Aperiodic Binary Invertible Sequences, IEEE Trans. Inf. Theory, 42, 1604 1612, 1996.

Sulzer, M. P.: A radar technique for high range resolution incoherent scatter autocorrelation function measurements utilizing the full average power of klystron radars, Radio Sci., 21, 1035-1040, 1986.

Turunen, T., Silén, J., Nygrén, T., and Jalonen, L.: Observations of a thin Es-layer by the EISCAT radar, Planet. Space Sci., 33, 1407-1416, 1985

Wannberg, G.: The G2-System and general purpose alternating code experiments for EISCAT, J. atmos. terr. Phys., 55, 543-557, 1993.

Widrow, B. and Stearns, S. D.: Adaptive signal processing, Upper Saddle River, NJ: Prentice-Hall, 1985.

Wen, C. H., Doherty, J. F., and Mathews, J. D.: Adaptive filtering for the separation of incoherent scatter and meteor signals for Arecibo observation data, J. Atmos. Terr. Phys., 67, 1190-1195, 2005. 